Ann. Biol. anim. Bioch. Biophys., I967, 7 (3), 281-293.

\title{
ÉVOLUTION SELON L'AGE ET LE POIDS DE LA COMPOSITION CORPORELLE DU RAT BLANC SOUMIS A UN RATIONNEMENT ENERGETIQUE APRÈS LE SEVRAGE
}

\author{
B. DESMOULIN \\ avec la collaboration technique de M. Lecourtier et Françoise Houlier \\ Station de Recherches sur l'Élevage des Porcs, \\ Centre national de Recherches zootechniques, 78 -Jouy-en-Josas \\ Institut national de la Recherche agronomique
}

SOMMAIRE

Une expérience a été réalisée sur des rats blanc de souche Wistar après le sevrage, dans le but d'étudier l'influence du niveau énergétique d'alimentation et de ses variations au cours de la croissance sur le gain de poids et la composition corporelle. A cet effet, les animaux soumis à un même apport azoté journalier reçoivent des apports énergétiques différents, Haut ou Bas, selon l'âge : Haut-Haut (H-H), Bas-Haut (B-H), Haut-Bas (H-B) et Bas-Bas (B-B); au niveau Bas, les rats sont restreints de 25 p. roo par rapport au niveau Haut, lui-même voisin de la consommation à volonté. Les abattages sont effectués à âges égaux et à poids égaux à ceux des témoins H-H.

Il ressort de cette étude que les rats restreints de façon continue (B-B) ont une croissance réduite de $18 \mathrm{p}$. 100. Les consommations et l'utilisation pratique de l'azote et de l'énergie montrent que ces animaux peuvent s'adapter à un apport énergétique restreint en limitant leurs dépenses d'énergie pour l'entretien ; mais l'efficacité azotée est abaissée par rapport à celle des animaux témoins. Lorsque la distribution d'énergie est accrue après un niveau Bas (B-H), l'efficacité azotée n'est pas modifiée, alors que l'efficacité énergétique est accrue durant la croissance compensatrice. Par contre, une réduction de l'apport d'énergie à la suite d'un niveau Haut (H-B) provoque une dépression de l'utilisation des éléments azotés et énergétiques.

Au même âge, mais à des poids différents, certaines différences de composition corporelle ont été observées. Après 3 semaines, les animaux restreints ont un poids inférieur à celui des témoins et la teneur en lipides de leur carcasse est plus faible. Après 6 semaines, les animaux du lot B-H ont une teneur en lipides supérieure à celle des animaux des lots $\mathrm{H}-\mathrm{B}$ et $\mathrm{B}-\mathrm{B}$, dont les poids sont plus faibles. Par contre, au même poids - de $15 \circ \mathrm{g}$ d'une part et de $25 \circ \mathrm{g}$ d'autre part - les compositions corporelles sont à peu près semblables, quel que soit le type de rationnement.

Dans nos conditions expérimentales, les rats sont en mesure de modifier l'utilisation de l'azote et de l'énergie selon les variations successives des niveaux énergétiques imposées durant la croissance. Les dépôts protéiques, qui sont prioritaires sur les dépôts lipidiques, ne sont pas influencés par la restriction; celle-ci ne limite par ailleurs le développement des dépôts lipidiques que durant le début de la croissance.

\section{INTRODUC'TION}

L'influence de la consommation de protéines et d'énergie sur le gain de poids et sa composition a fait 1'objet de très nombreuses recherches : chez le Rat (BosshaRDT et Barnes, I946 ; Forbes, I955; Alisison, I956), chez le Porc (PoND, I960 ; CLAW- 
sON, I962 ; WAGNER et ClaRk, I963; WALLACE, I964; ROBINSON et al., I964; HENRY et RÉRAT, I964 ; BowLAND, I963 ; AuMAITRE et al., I964), chez le Poulet (GuIl_AUME, I96I ; Summers et al., I965). L'efficacité nutritionnelle durant la croissance peut, selon l'espèce animale considérée, être différente pour des rations semblables (MEYER et NELson, I963; LIKUSKI et BowlaND, I96I ; KuRYvial et al., Ig62). Toutefois, chez les différentes espèces, l'ingestion élevée de protéines et d'énergie modifie peu les dépôts protéiques de croissance, alors que l'ingestion élevée d'énergie favorise l'excès des dépôts lipidiques dans le croît.

Selon des méthodes distinctes, ces observations ont été effectuées, soit en modifiant le rapport $\frac{\text { Principes Énergétiques }}{\text { Principes azotés }}$ de l'aliment, soit en réduisant le niveau de sa distribution.

I. La variation du rapport Principes Énergétiques/Principes Azotés de l'aliment, obtenue en modifiant les taux énergétique ou azoté, s'accompagne parfois de différences qualitatives (glucides, lipides, acides aminés) qui retentissent sur l'appétit (CALET, Ig6r). La composition du croît peut ainsi résulter d'influences qualitatives et quantitatives non différenciées.

2. La réduction globale et sévère du niveau d'alimentation entraîne, par ailleurs, sur la composition du croît)des effets que les études de McMEEKAN (I940-I94I) chez le Porc ont amplement démontrés : au même poids final de $90 \mathrm{~kg}$, mais à des âges parfois différents, la composition du gain de poids dépend de la succession des niveaux alimentaires auxquels les porcs ont été soumis durant la croissance. HamMOND interprète cette observation en fonction d'un ordre de priorité dans la formation des tissus selon l'âge. Les résultats de McMEEKAN indiquent nettement les difficultés de 1'obtention simultanée d'une croissance satisfaisante et d'une faible adiposité par la méthode du rationnement plimentaire. Toutefois, ils ne distinguent pas l'influence de la restriction énergétiqua yé la restriction en azote.

L'expérience suivante a été effectuée chez le Rat blanc après le sevrage : à ingérés de protéines, et d'acides aminés égaux selon l'âge, une différence des niveaux énergétiques d'alimentation est obtenue en associant une réduction du niveau d'alimentation à une augmentation proportionnelle du taux azoté de l'aliment. Les influences sur lacomposition corporelle et 1'efficacité nutritionnelle sont étudiées par la méthode des abattages successifs. Le schéma expérimental de McMEEKAN chez leporc est utilisé en effectuant, à deux stades de, la croissance du rat, deux séries d'abattages selon 1'âge et le poids des animaux X'́moins.

\section{MATÉRIEL ET METHODES}

\section{I - Animaux - Période pré-expérimentale}

Ioo rats mâles de souche Wistar ont été choisis au Centre de Gif-sur-Yvette d'après les caractéristiques suivantes d'homogénéité au sevrage :

$$
\text { Age : 22-23 jours. Poids : } 40 \text { à } 4^{8} \mathrm{~g} \text {. }
$$


Ces animaux reçoivent, durant une période de 6-7 jours destinée à contrôler leurs potentialités de consommation et de croissance, un régime pré-expérimental ( ${ }^{(1)}$ équilibré et distribué à volonté. $\mathrm{Au}$ terme de cette période, les rats sont âgés de 4 semaines et pèsent $65 \pm 5 \mathrm{~g}$.

(') Caséine : 120. Cystine : 3. Amidon : 527. Saccharose : 200. Huile d'arachide : 80. Cellulose : 20. Mélange salin : 40. Mélange vitaminique : Io.

\section{II - Régimes expérimentaux - Méthode de rationnement}

Le niveau de restriction étudié est de $25 \mathrm{p}$. Ioo inférieur au niveau témoin de distribution. Pour égaliser l'ingestion journalière d'azote aux deux niveaux d'alimentation, deux régimes sont nécessaires :

. un régime à I2 p. Ioo de protéines distribué aux animaux alimentés au niveau Haut. . un régime à $16 \mathrm{p}$. Ioo de protéines distribué aux animaux alimentés au niveau Bas.

\begin{tabular}{|c|c|c|}
\hline $\begin{array}{l}\text { Composition des régimes } \\
\qquad(\mathrm{g} / \mathrm{kg})\end{array}$ & Haut & Bas \\
\hline 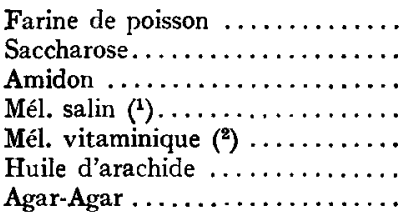 & $\begin{array}{l}174,6 \\
215 \\
460,4 \\
40 \\
10 \\
80 \\
20\end{array}$ & $\begin{array}{l}232,8 \\
215 \\
402,2 \\
40 \\
10 \\
80 \\
20\end{array}$ \\
\hline
\end{tabular}

(2) OSBORNE et MENDEL.

(2) Rerat A., Février C., Henry Y., Lougnon J., 1964.

Pour éviter les irrégularités de consommations et de croissance observées avec un régime distribué à volonté, il a été nécessaire d'appliquer une échelle d'alimentation établie dans une préexpérience, grâce à la définition d'une courbe moyenne de consommation. Cette échelle d'alimentation représente au niveau Témoin une rédusion moyenne de ro $\mathrm{p}$. roo par rapport au niveau spontané de la consommation du même réginfé.

Dans ces conditions, le niveau restreint est inférieur de $25 \mathrm{p}$. roo à ce niveau témoín et les quantités. distribuées à chacun des niveaux sont consommées sans cumuler les refus. Ce mode de distribution des régimes suivant deux échelles de rationnement journalier est représenté isur la figure $I$.

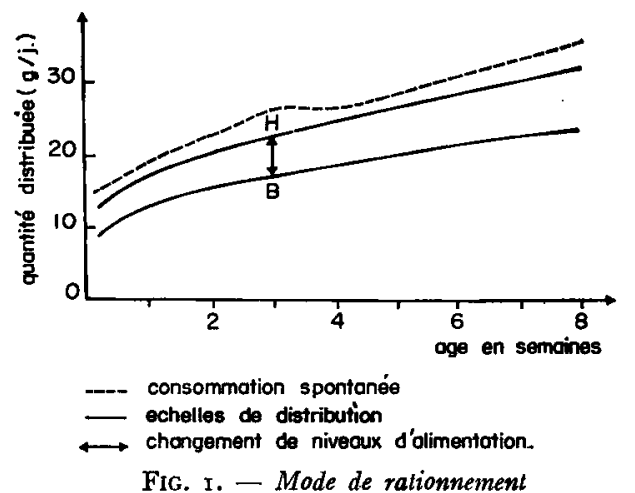




\section{III - Schéma expérimental - Mode d'abattage}

Durant deux périodes successives de 3 semaines, les animaux sont soumis à l'un ou l'autre des niveaux d'alimentation selon le schéma suivant :

Lot Témoin.$\ldots \ldots \ldots \ldots \ldots \ldots \ldots \ldots \ldots \ldots \ldots$ Haut-Haut $(\mathrm{H}-\mathrm{H})$

Lot Restreint de façon continue ........... Bas-Bas (B-B)

Lots Restreints de façon discontinue ......... Haut-Bas (H-B) et Bas-Haut (B-H)

Deux séries d'abattages sont effectuées :

à âge égal au terme de chaque période expérimentale

- après 3 semaines - après 6 semaines

à poids égal à celui des animaux témoins en fin de période

- à $150 \mathrm{~g}$

- à $250 \mathrm{~g}$.

La figure 2 représente les courbes de croissance et les stades d'abattage dans les 1o lots qui ont été constitués d'après la relation poids final - croît de la période préexpérimentale.

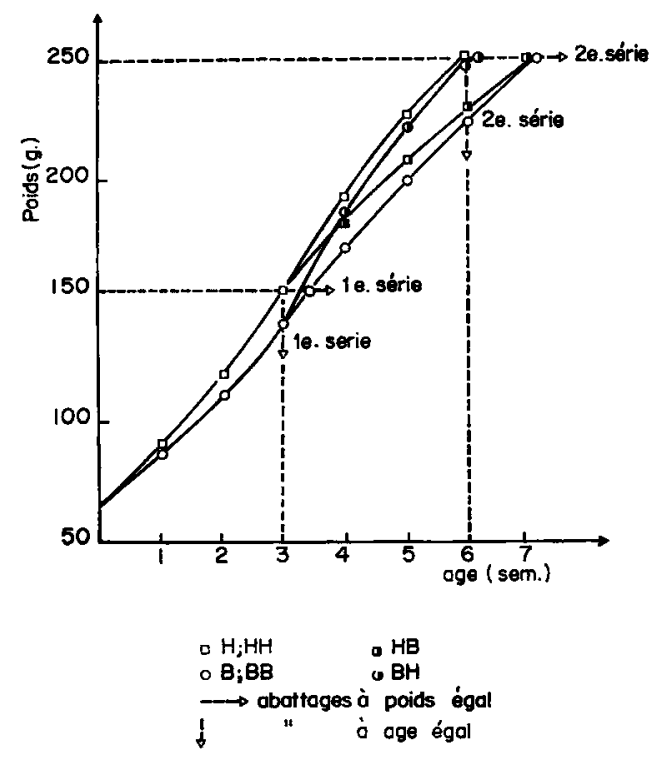

FIG. 2. - Courbe de croissance et mode d'abaltage

\section{IV - Composition corporelle - Méthodes d'analyse}

Les carcasses entières, préalablement stockées en chambre froide $\left(-15^{\circ} \mathrm{C}\right)$, sont coupées en morceaux et autoclavées pendant 20 minutes à $120^{\circ} \mathrm{C}$ (MICKEISEN, 1959). Après addition d'un poids connu d'eau, elles sont homogénéisées à l'aide d'un mixer ultraturrax.

Des parties aliquotes sont prélevées en vue du dosage d'azote par la méthode Kjeldahl - de la détermination de la matière sèche à l'étuve à $103^{\circ}$ - des cendres après calcination durant $3^{6}$ heures au four à $55^{\circ}$. Les teneurs en lipides sont estimées par la différence : matière sèche - (protéines + cendres).

Les résultats de l'analyse corporelle permettent le calcul du coefficient de rétention de l'azote 
et de l'énergie, analogue au coefficient d'utilisation pratique (CUP) selon TERroine et Valla (I933).

$$
\begin{gathered}
\text { CUPazote }=\frac{\mathrm{N} \text { retenu dans les tissus }(\mathrm{g}) \times 100}{\mathrm{~N} \text { ingéré }(\mathrm{g})} \\
\text { CUPénergie }=\frac{\text { Énergie retenue dans les tissus }(\mathrm{kcal}) \times 100}{\text { Energie ingérée }(\mathrm{kcal})}
\end{gathered}
$$

Les teneurs initiales en azote et en énergie des carcasses sont obtenues à partir d'équations de régression des constituants corporels (lipides et protéines) sur le poids vif (RtERAT et al., 1964). Les teneurs finales en énergie sont calculées en utilisant les coefficients d'ATwATER et BRYANT par la somme Lipides finaux $\times 9,40+$ Protéines finales $\times 5,65$.

L'interprétation statistique est effectuée en testant l'homogénéité des variances entre lots, puis en comparant les moyennes (test de KEvis). Par ailleurs, pour un même mode d'abattage, le schéma factoriel entre les lots $\mathrm{H}-\mathrm{H}, \mathrm{B}-\mathrm{H}, \mathrm{H}-\mathrm{B}$ et B-B permet d'analyser l'influence du niveau d'alimentation selon la période d'application.

\section{RÉSULTATS}

L'influence $d u$ rationnement énergétique sur la consommation, la croissance et la composition corporelle est présentée dans les tableaux I, 2 et 3. Les différents lots sont considérés selon les références d'âge ou de poids à l'abattage.

\section{I - Consommation}

Dans le tableau I, les consommations d'énergie et de protéines sont exprimées d'une part en quantités absolues, d'autre part en quantités relatives à celles des animaux témoins.

Les quantités rapportées dans chaque lot sont celles imposées par le rationnement journalier à chaque animal du lot durant toute sa croissance. Selon la référence expérimentale d'abattage, on peut remarquer :

r. A âge égal : les animaux restreints de façon continue ont consommé selon le mode de rationnement 75 p. Ioo de l'énergie distribuée au niveau élevé d'alimentation. Dans les lots B-H et $\mathrm{H}-\mathrm{B}$, dont les niveaux ont été inversés après 3 semaines, la consommation d'énergie est comparable après 6 semaines : elle représente dans les lots $\mathrm{B}-\mathrm{H}$ et $\mathrm{H}-\mathrm{B}$ respectivement $90 \mathrm{p}$. Ioo et $86 \mathrm{p}$. Ioo de l'énergie consommée dans le lot témoin $\mathrm{H}-\mathrm{H}$.

Dans tous les lots, les consommations de protéines sont égales.

2. A poids égal : tous les animaux, excepté ceux du lot B-H, ont des consommations totales d'énergie comparables à celles des animaux témoins. Au poids de $25^{\circ} \mathrm{g}$, la consommation d'énergie dans le lot B-H est inférieure de Io p. Ioo à celle des témoins. Par ailleurs, dans le lot $\mathrm{H}-\mathrm{B}$, elle est supérieure de Io $\mathrm{p}$. Ioo à celle des animaux du lot B-B.

Comme l'ingestion journalière d'azote estégale dans tous les lots, les consommations totales de protéines augmentent avec la longueur de la période de croissance. A I5o g, on remarque dans le lot $\mathrm{B}$ une consommation supérieure de $24 \mathrm{p}$. Ioo à celle du lot $\mathrm{H}$. 
A $250 \mathrm{~g}$, dans les lots $\mathrm{H}-\mathrm{B}$ et $\mathrm{B}-\mathrm{B}$, les consommations totales de protéines sont plus élevées de $28 \mathrm{p}$. Ioo et $23 \mathrm{p}$. Ioo respectivement que dans le lot $\mathrm{H}-\mathrm{H}$.

Tous les animaux restreints, sauf ceux du lot B-H, ont donc une consommation de protéines accrue en moyenne de $25 \mathrm{p}$. Ioo par rapport à celle des témoins de même poids.

\section{TABLEAU I}

Quantités d'énergie de protéines ingérées selon les niveaux d'alimentation et le mode d'abattage.

\begin{tabular}{|c|c|c|c|c|c|c|}
\hline \multirow{2}{*}{ Abattage à âge égal } & \multicolumn{2}{|c|}{3 semaines } & \multicolumn{4}{|c|}{6 semaines } \\
\hline & Haut & Bas & Haut-Haut & Bas-Haut & Haut-Bas & Bas-Bas \\
\hline $\begin{array}{l}\text { Quantités } \\
\text { absolues }\end{array}\left\{\begin{array}{l}\text { Énergie }(\mathrm{kcal}) \ldots \ldots \\
\text { Protéines }(\mathrm{g}) . . . \ldots \ldots\end{array}\right.$ & $\begin{array}{r}1175 \\
\quad 34,7\end{array}$ & $\begin{array}{l}894 \\
34,0\end{array}$ & $\begin{array}{l}2785 \\
\quad 82,2\end{array}$ & $\begin{array}{l}2504 \\
81,5\end{array}$ & $\begin{array}{l}2397 \\
81,2\end{array}$ & $\begin{array}{l}2116 \\
\quad 80,5\end{array}$ \\
\hline $\begin{array}{l}\text { Quantités } \\
\text { relatives }\end{array}\left\{\begin{array}{l}\text { Énergie } \ldots \ldots \ldots \ldots \\
\text { Protéines } \ldots \ldots \ldots \ldots\end{array} \mid\right.$ & $\begin{array}{l}100 \\
100\end{array}$ & $\begin{array}{l}76 \\
98\end{array}$ & $\begin{array}{l}100 \\
100\end{array}$ & $\begin{array}{l}90 \\
99\end{array}$ & $\begin{array}{l}86 \\
99\end{array}$ & $\begin{array}{l}76 \\
98\end{array}$ \\
\hline $\begin{array}{l}\text { Poids : déficit en valeur absolue } \\
\text { à l'abattage }(\mathrm{g}) \ldots \ldots \ldots \ldots \ldots\end{array}$ & $\mathbf{0}$ & -16 & 0 & -6 & -23 & -26 \\
\hline \multirow{2}{*}{ Abattage à poids égal } & \multicolumn{2}{|c|}{$150 \mathrm{~g}\left({ }^{1}\right)$} & \multicolumn{4}{|c|}{$250 \mathrm{~g} \mathrm{(})$} \\
\hline & Haut & Bas & Haut-Haut & Bas-Haut & Haut-Bas & Bas-Bas \\
\hline $\begin{array}{l}\text { Quantités } \\
\text { absolues }\end{array}\left\{\begin{array}{l}\text { Énergie (kcal) ...... } \\
\text { Protéines (g)....... }\end{array}\right.$ & $\begin{array}{l}1175 \\
\quad 34,7\end{array}$ & $\begin{array}{r}1110 \\
\quad 43,0\end{array}$ & $\begin{array}{l}2785 \\
\quad 82,2\end{array}$ & $\begin{array}{l}2522 \\
82,5\end{array}$ & $\begin{array}{l}2863 \\
105,0\end{array}$ & $\begin{array}{l}2650 \\
100,9\end{array}$ \\
\hline $\begin{array}{l}\text { Quantités } \\
\text { relatives }\end{array}\left|\begin{array}{l}\text { Énergie } \ldots \ldots \ldots \ldots \\
\text { Protéines } \ldots \ldots \ldots \ldots\end{array}\right|$ & $\begin{array}{l}100 \\
100\end{array}$ & $\begin{array}{r}97 \\
124\end{array}$ & $\begin{array}{l}100 \\
100\end{array}$ & $\begin{array}{r}91 \\
100\end{array}$ & $\begin{array}{l}105 \\
128\end{array}$ & $\begin{array}{r}96 \\
123\end{array}$ \\
\hline Age supplém. à l'abattage (j).. & 0 & +4 & 0 & +1 & +8 & +8 \\
\hline
\end{tabular}

(1) Écart type $\mathrm{S} \overrightarrow{\mathrm{x}}= \pm 3 \mathrm{~g}$.

\section{II - Croissance. Efficacité alimentaire}

Sur le tableau 2, on note que la restriction énergétique de $25 \mathrm{p}$. Ioo réduit de I $8 \mathrm{p}$. Ioo la vitesse de croissance après 3 semaines. Cette réduction se retrouve après 6 semaines lorsque la différence des niveaux d'alimentation est maintenue. Par contre, elle disparaît lorsque les animaux sont soumis à un niveau identique d'alimentation (Haut ou Bas) durant la deuxième période de croissance. L'interprétation du schéma factoriel H-H, B-H, H-B et B-B indique un effet significatif du niveau d'alimentation seulement en deuxième période sur la vitesse de croissance. Au poids final de $250 \mathrm{~g}$, ce résultat se traduit par les abattages simultanés dans les lots $\mathrm{H}-\mathrm{H}$ et $\mathrm{B}-\mathrm{H}$ après 42 jours d'une part, dans les lots $\mathrm{H}-\mathrm{B}$ et B-B après 50 jours d'autre part. 
Les variations de la vitesse de croissance sont très accusées au changement de niveaux d'alimentation. Comme ce changement est appliqué sans tenir compte des écarts de poids entre les animaux $\mathrm{H}$ et $\mathrm{B}$ après 3 semaines, les croissances observées ont été comparées entre les lots dont les consommations journalières sont égalisées durant la deuxième période : $\mathrm{H}-\mathrm{H}$ et $\mathrm{B}-\mathrm{H}$ d'une part, $\mathrm{H}-\mathrm{B}$ et $\mathrm{B}-\mathrm{B}$ d'autre part.

\section{'ABLEAU 2}

Vitesse de croissance $(\mathrm{g} / \mathrm{j})$ selon les niveaux d'alimentation et la période d'application.

\begin{tabular}{|c|c|c|c|c|c|c|}
\hline \multirow{2}{*}{ Abattage à âge égal } & \multicolumn{2}{|c|}{3 semaines } & \multicolumn{4}{|c|}{6 semaines } \\
\hline & Haut & Bas & Haut-Haut & Bas-Haut & Haut-Bas & Bas-Bas \\
\hline \multicolumn{7}{|l|}{ Croissance $(\mathrm{g} / \mathrm{j})$ : } \\
\hline $1^{\text {re }}$ période....... & \multirow[t]{3}{*}{4,36} & \multirow[t]{2}{*}{3,56} & 4,35 & \multirow{2}{*}{$\frac{5,48}{5,09}$} & \multirow{2}{*}{$\begin{array}{l}4,26 \\
3,54\end{array}$} & \multirow{2}{*}{$\frac{3,54}{4,03}$} \\
\hline $2^{\circ}$ période............... & & & 4,56 & & & \\
\hline Total périodes.............. & & \multirow[b]{2}{*}{141,0} & 4,46 & $\overline{4,16}$ & 3,78 & $\overline{3,74}$ \\
\hline Poids final $(g) \ldots \ldots \ldots \ldots \ldots$ & 156,8 & & $25 \overline{2,7}$ & 246,4 & 229,4 & 225,2 \\
\hline \multirow{2}{*}{ Abattage à poids égal } & \multicolumn{2}{|c|}{$150 \mathrm{~g}$} & \multicolumn{4}{|c|}{$250 \mathrm{~g}$} \\
\hline & Haut & Bas & Haut-Haut & Bas-Haut & Haut-Bas & Bas-Bas \\
\hline \multicolumn{7}{|l|}{ Croissance $(g / j)$ : } \\
\hline 10 période.... & 4,36 & 3,80 & 4,35 & 3,57 & 4,10 & 3,50 \\
\hline $2^{\mathbf{e}}$ période.... & & & 4,56 & 5,12 & 3,64 & $\overline{3,93}$ \\
\hline Total périodes.............. & & & 4,46 & 4,40 & 3,82 & 3,75 \\
\hline $\begin{array}{l}\text { Durée des périodes ou âge expé- } \\
\text { rimental }(j) \ldots \ldots \ldots \ldots \ldots \ldots\end{array}$ & 21 & 24-25 & 42 & $42-43$ & $49-50$ & 50 \\
\hline
\end{tabular}

Les moyennes reliées par un trait discontinu sont significativement différentes au seuil 0,05 .

Dans le lot B-H, la croissance est plus élevée de 16 p. Ioo que celle du lot H-H.

Dans le lot H-B, la croissance est plus faible de 7 p. Ioo que celle du lot B-B.

Durant la deuxième période de la croissance, les animaux du lot $\mathrm{B}-\mathrm{H}$ rattrapent rapidement le retard de croissance que présentent les animaux du lot B à 3 semaines. Ceux du lot $\mathrm{H}-\mathrm{B}$ perdent lentement l'avantage de croissance présenté par les animaux $H$ à 3 semaines.

\section{III - Composition corporelle - Rétentions absolues d'énergie et d'azote.}

Dans le tableau 3, les teneurs en différents constituants corporels sont présentées pour chaque lot, selon le mode d'abattage. 
I. A âge égal certaines différences de composition corporelle sont remarquées - à 3 semaines, les animaux du lot B ont une teneur en lipides plus faible que celle du lot $\mathrm{H}$ - à 6 semaines, dans le lot $\mathrm{B}-\mathrm{H}$, la teneur en lipides est plus élevée que dans les autres lots.

2. A poids égal, quel que soit le mode de rationnement adopté, les animaux ont des compositions corporelles à peu près semblables, à I $50 \mathrm{~g}$ d'une part, à $25^{\circ} \mathrm{g}$ d'autre part.

TABLEAU 3

Composition chimique corporelle ( $p .100$ du poids frais)

Efficacité des rétentions d'azote et d'énergie ( $p .100$ des quantités ingérées)

\begin{tabular}{|c|c|c|c|c|c|c|c|}
\hline \multirow{2}{*}{\multicolumn{2}{|c|}{ Abattage à âge égal }} & \multicolumn{2}{|c|}{3 semaines } & \multicolumn{4}{|c|}{6 semaines } \\
\hline & & Haut & Bas & Haut-Haut & Bas-Haut & Haut-Bas & Bas-Bas \\
\hline \multirow[t]{2}{*}{$\begin{array}{l}\text { Composition } \\
\text { corporelle }\end{array}$} & $\left\{\begin{array}{l}\text { Eau }(\%) \ldots . . \\
\text { Cendres } \% \ldots \\
\text { Protéines } \%\end{array}\right.$ & $\begin{array}{r}66,8 \\
3,0 \\
17,7 \\
\end{array}$ & $\begin{array}{r}69,0 \\
3,1 \\
18,9 \\
\end{array}$ & $\begin{array}{r}64,9 \\
3,2 \\
18,8 \\
\end{array}$ & $\begin{array}{r}63,3 \\
3,1 \\
18,0 \\
\end{array}$ & $\begin{array}{r}64,3 \\
3,2 \\
18,9 \\
\end{array}$ & $\begin{array}{r}64,2 \\
3,2 \\
18,9 \\
\end{array}$ \\
\hline & Lipides $\% \ldots$ & $\overline{12,5}$ & 9,0 & $\overline{13,1}$ & 15,6 & 13,6 & 13,8 \\
\hline \multirow{2}{*}{ CUP } & Azote ........ & 43,9 & $4 \overline{0,8}$ & 42,5 & $\overline{38,7}$ & 38,1 & 37,4 \\
\hline & Énergie...... & $\underline{17,5}$ & $\overline{15,1}$ & 16,5 & 19,4 & 16,9 & 17,5 \\
\hline \multirow{2}{*}{\multicolumn{2}{|c|}{ Abattage à poids égal }} & \multicolumn{2}{|c|}{$150 \mathrm{~g}$} & \multicolumn{4}{|c|}{$250 \mathrm{~g}$} \\
\hline & & Haut & Bas & Haut-Haut & Bas-Haut & Haut-Bas & Bas-Bas \\
\hline \multirow{4}{*}{$\begin{array}{l}\text { Composition } \\
\text { Corporelle }\end{array}$} & Eau $\% \ldots$ & 66,8 & 68,2 & 64,9 & 63,7 & 63,7 & 64,2 \\
\hline & Cendres $\% \ldots$. & 3,0 & 3,1 & 3,1 & 3,1 & 3,2 & 3,3 \\
\hline & Protéines $\% \ldots$ & 17,7 & 18,5 & 18,8 & 18,4 & 19,1 & 19,4 \\
\hline & Lipides $\%$ & 12,5 & 10,2 & $\overline{13,1}$ & 14,8 & 14,0 & 13,1 \\
\hline \multirow{2}{*}{ CUP } & Azote..... & 43,9 & 39,5 & $\overline{42,5}$ & 41,0 & 35,7 & 35,9 \\
\hline & Énergie...... & 17,5 & $\overline{16,5}$ & $\overline{16,5}$ & 18,9 & $\overline{16,2}$ & 16,8 \\
\hline
\end{tabular}

Les moyennes reliées par un trait discontinu sont significativement différentes au seuil 0,05 .

Sur les figures (3) les constituants énergétiques corporels (protéines et lipides) ont été séparés de façon à préciser l'influence stricte de la restriction en énergie sur les quantités de lipides déposées.

A I50 g, les animaux du lot B ont retenu $149 \mathrm{kcal}$ lipidiques pour $178 \mathrm{kcal}$ dans le lot $\mathrm{H}$ au même poids. Cette différence n'est pas significative, alors qu'à 3 semaines la rétention de II4 $\mathrm{kcal}$ lipidiques dans le lot B est inférieure à celle des animaux du lot $\mathrm{H}$. Ainsi, les animaux restreints en énergie sont, à âge égal, moins riches en lipides mais leur poids est plus faible.

A $25^{\circ} \mathrm{g}$, les rétentions énergétiques sont égales dans les lots $\mathrm{B}-\mathrm{H}$ et $\mathrm{H}-\mathrm{B}$ d'une part, dans les lots $\mathrm{H}-\mathrm{H}$ et $\mathrm{B}-\mathrm{B}$ d'autre part. Les fractions lipidiques ne sont pas 
significativement différentes entre ces quatre lots. Toutefois, à 6 semaines, la rétention de $353 \mathrm{kcal}$ lipidiques dans le lot B-H est plus élevée que dans les lots $\mathrm{H}-\mathrm{H}$, $\mathrm{B}-\mathrm{B}$ et $\mathrm{H}-\mathrm{B}$ qui ont retenu également en moyenne $300 \mathrm{kcal}$ lipidiques. Mais les animaux des lots B-B et $\mathrm{H}-\mathrm{B}$ sont, à âge égal, d'un poids plus faible que ceux des lots B-H et $\mathrm{H}-\mathrm{H}$.

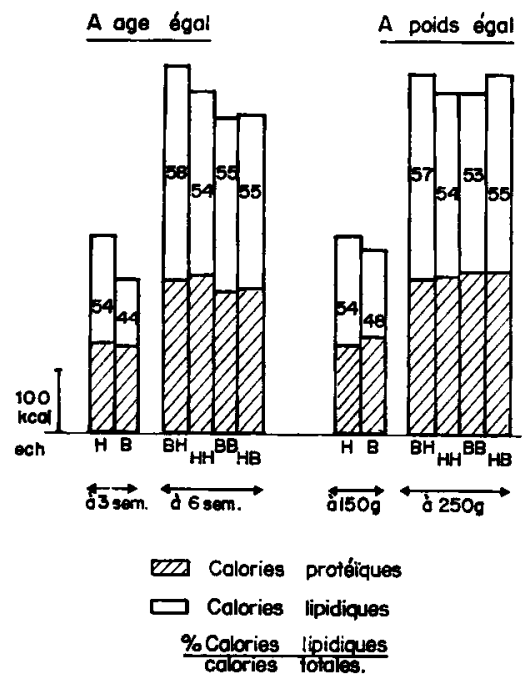

FIG. 3. - Constituants énergetiques corporels

L'évolution des dépôts énergétiques selon l'âge et le poids est par ailleurs présentée sur cette figure (3).

En début de croissance, les lipides représentent 44 à 48 p. 1oo des constituants énergétiques dans les lots $B$ restreints et $54 \mathrm{p}$. Ioo dans les lots $\mathrm{H}$ témoins. En fin de croissance, les lipides représentent une fraction énergétique à peu prés égale dans tous les lots. Durant la $2^{\mathrm{e}}$ période, les lipides se sont déposés en quantités très importantes dans les lots B-H et B-B, alors que les dépôts sont restés constants dans les lots $\mathrm{H}-\mathrm{H}$ et $\mathrm{H}-\mathrm{B}$. Ainsi, le développement des tissus lipidiques en fin de croissance est d'autant plus influencé par les niveaux d'alimentation que la restrictions'applique tôt après le sevrage.

\section{IV - Efficacité des rétentions}

Les résultats concernant l'efficacité de la rétention azotée et énergétique (CUP) apparaissent dans le tableau 3. Selon le mode d'application du rationnement, on peut remarquer :

I. Dans les lots B et B-B, l'efficacité azotée est réduite par rapport à celle obtenue dans les lots $\mathrm{H}$ et $\mathrm{H}-\mathrm{H}$. L a réduction apparaît d'autant plus accusée que la restriction énergétique est plus longtemps appliquée si l'on considère les différences à poids égal. Par contre, l'efficacité énergétique n'est pas modifiée, malgré la restriction de 25 p. roo à âge égal, ou l'augmentation de la durée d'entretien à poids égal.

2. Après changement de niveau, l'efficacité azotée est comparable dans les lots B-H et $\mathrm{H}-\mathrm{H}$ d'une part, dans les lots H-B et B-B d'autre part. Compte tenu 
des résultats obtenus dans les lots $\mathrm{H}$ et $\mathrm{B}, 1$ 'évolution de l'efficacité azotée est en accord avec les observations effectuées précédemment sur la vitesse de croissance : dans le lot $\mathrm{B}-\mathrm{H}$, elle a été améliorée et dans le lot $\mathrm{H}-\mathrm{B}$ elle a été réduite en fin de croissance. D'autre part, l'efficacité énergétique est finalement plus élevée dans le lot $\mathrm{B}-\mathrm{H}$ que dans les lots $\mathrm{H}-\mathrm{H}, \mathrm{B}-\mathrm{B}$ et $\mathrm{H}-\mathrm{B}$ où elle est comparable.

\section{DISCUSSION}

Chez le Rat blanc, soumis après sevrage à une restriction énergétique de $25 \mathrm{p}$. Ioo, sans modification de 1'apport azoté journalier, une composition corporelle sembable peut être obtenue par des voies différentes. Comment interpréter ce résultat lorsque les influences de la restriction énergétique sur la vitesse de croissance et l'efficacité alimentaire sont considérées?

I. La restriction continue réduit de $\mathrm{I} 8 \mathrm{p}$. Ioo la vitesse de croissance. Ainsi, à poids d'abattage égal, dans les lots restreints, l'allongement de la période de croissance par rapport aux lots témoins entraîne une augmentation de la consommation totale de protéines sans toutefois modifier la consommation totale d'énergie. L'efficacité azotée est abaissée sans que l'efficacité énergétique soit modifiée dans les lots restreints. Toutefois, les dépôts de protéines et de lipides ne diffèrent pas en fin de croissance; plusieurs hypothèses peuvent donc être émises :

- La restriction énergétique entraîne une dépense d'azote à des fins énergétiques (ForBEs, I955).

- Une limitation des dépenses énergétiques d'entretien peut être envisagée. Pour ung même gain de poids, mais une durée d'entretien plus longue de 8 jours dans le lot restreint, les animaux B-B et $\mathrm{H}-\mathrm{H}$ ont consommé et retenu des quantités égales d'énergie. MORIN-JOMAIN (Ig62) soumettant des rats à des restrictions alimentaires discontinues, a observé par la méthode des bilans respiratoires l'entraînement des animaux à diminuer leurs dépenses énergétiques. Cette observation, effectuée également chez des animaux restreints en énergie de façon continue, pourrait donc s'appliquer aux dépenses d'entretien, puisque les animaux ont une même composition corporelle au même poids que les témoins.

En définitive, les " dépenses totales " d'énergie et d'azote sont ajustées par l'animal au niveau métabolique permis par les apports alimentaires d'énergie.

2. La restriction discontinue est appliquée en égalisant après 3 semaines les consommations entre les lots $\mathrm{H}-\mathrm{H}$ et $\mathrm{B}-\mathrm{H}$ d'une part et entre les lots B-B et H-B d'autre part. L'apport azoté étant ainsi maintenu constant, les modifications de la croissance et de l'efficacité alimentaire trađuisent donc les réponses aux seuls changements de niveau énergétique, sans interférence de 1'appétit.

a) Un niveau Bas, suivi d'un niveau Haut entraîne une "croissance compensatrice " (BoHmaN, I955). Or, ce changement peut, à lui seul, modifier le niveau de consommation et la composition corporelle (WILSON-OsBOURN, I960) ; lorsque la consommation est égalisée après des niveaux d'alimentation $\mathbf{B}$ et $\mathbf{H}$ différents, une compensation de croissance et une augmentation des dépôts lipidiques sont ob- 
servées dans le lot $\mathrm{B}-\mathrm{H}$ par rapport au lot $\mathrm{H}$-H. L'efficacité énergétique accrue dans le lot B-H confirmerait l'hypothèse d'une adaptation des animaux à épargner l'énergie lorsque celle-ci a été limitée au niveau Bas. Comme les animaux du lot B-H déposent plus de lipides que ceux du lot témoin $\mathrm{H}-\mathrm{H}$, l'épargne d'énergie porterait sur l'entretien, cette épargne pourrait s'accompagner d'une diminution relative du coût des đépôts lipidiques par rapport au coût des dépôts protéiques durant la croissance compensatrice.

b) Un niveau Haut, suivi d'un niveau Bas entraîne un " freinage de croissance » (FÉvRIER, I959). Ainsi, à consommation égalisée après des niveaux $\mathrm{H}$ et $\mathrm{B}$ différents, les animaux du lot $\mathrm{H}-\mathrm{B}$ perdent progressivement sur ceux du lot B-B l'avantage de poids acquis à 3 semaines au niveau $H$. L'efficacité alimentaire et la composition corporelle ne diffèrent pas dans les lots H-B et B-B au même poids final. Toutefois, les différences notées au terme de chaque période de croissance indiquent que les animaux du lot $\mathrm{H}-\mathrm{B}$ ont déposé la majorité de leurs lipides en première période, alors que les animaux du lot B-B ont effectué ces dépôts principalement en $2^{e}$ période. Contrairement aux différences observées par McMEEKan chez le Porc, les rats restreints en énergie sans restriction d'azote, ont une composition corporelle comparable aux rats témoins à $250 \mathrm{~g}$. Ceci peut être dû à des différences interspécifiques de maturité relative (BRODY, I945), mais également aux différences des conditions d'étude. La sévérité du rationnement et la durée des périodes de restriction peuvent conditionner très fortement l'évolution de la composition des dépôts de croissance.

En définitive, quel que soit le mode de rationnement adopté, les dépôts protéiques et lipidiques sont comparables pour un poids égal de $250 \mathrm{~g}$. Ce poids correspond environ à 40 p. roo de celui du rat adulte. En outre, la restriction énergétique ne modifie que l'évolution des dépôts lipidiques au cours des phases successives de la croissance. Dans les conditions de cette étude, les dépôts lipidiques sont réduits durant la première période de 3 semaines qui suit le sevrage, chez les animaux primitivement restreints. A l'issue de cette période, ils prennent une importance croissante avec l'âge. Il semble donc qu'une restriction plus sévère soit nécessaire pour limiter leur développement tardif.

Rę̧u pour publication en avril 1967.

\title{
SUMMARY
}

\author{
CHANGES ACCORDING TO AGE AND WEIGHT IN BODY COMPOSITION \\ OF WHITE RATS GIVEN RESTRICTED AMOUNTS OF ENERGY AFTER WEANING
}

During two successive periods of 3 weeks white rats of the Wistar strain were given, according to age, the same amount of protein but different amounts of energy : H-H, B-H, H-B and B-B. On the low intake of energy $(B)$ the diet had 16 per cent protein and on the high $(\mathrm{H})$ it had $\mathrm{I} 2$ per cent. The restriction of feed for the low intake of energy was to 25 per cent less than on the high intake, which was near to the maximum voluntary intake. In each group two series of killings were made according to the age and weight of the controls.

Restriction of energy reduced growth rate of groups B-B and H-B by 18 per cent compared with groups $\mathrm{H}-\mathrm{H}$ and $\mathrm{B}-\mathrm{H}$. Efficiency of protein utilization was 25 per cent less in the former two groups. Energetic efficiency was improved in group B-H compared with all other groups, among which there was no difference. In these conditions adaptation of animals to feed restricted in energy took the 
form of an adjustment of total expenditure of protein and energy during successive periods of growth. The effect of rationing on composition of weight gained was essentially to modify the development of deposition of lipids in each group. At the beginning of growth the deposited tissue was 44 to 48 per cent of the energy-supplying constituents in the B, restricted, groups, and 54 per cent in the $\mathrm{H}$, control, groups. At the end of growth lipids represented about the same proportion of energy in all groups. Thus the animals in groups B-B and B-H laid down large amounts of lipids in the second period while those in groups $\mathrm{H}-\mathrm{H}$ and $\mathrm{H}-\mathrm{B}$ made these deposits in equal amounts during each period. It seems then that a more severe restriction during the last stages of growth would be necessary to limit the late development of deposition of lipids.

\section{RÉFÉRENCES BIBLIOGRAPHIQUES}

Allison J. B., 1957. Calories and Protein Nutrition. Ann. New York Acad. of Sci., 69, 1009-1024.

Atwater W., Obryant A. P., 1903. The chemical composition of American food materials. U.S. Dept. Agric. Bull. 28.

Aumaitre A., Jouandet C., Salmon-Legagneur E., I964. Effets des taux énergétiques et protidiques de la ration' sur l'efficacité alimentaire et sur la croissance chez le porcelet. Ann. Zoolech., 13, 24I-253.

Bohman V. R., 1966. Compensatory growth of beef cattle, the effect of hay maturity. J. anim. Sci., 14, $249-255$.

BoHMan - V. R., TORREL C., I956. Compensatory growth of beef cattle; the effect of protein supplements. J. anim. Sci., 15, 1089-1096.

Bowland J. P., Sibbald I. R., Berg R. T., Hussar N., I958. Influence of dietary fat on energy consumption and digestion and on nitrogen utilization of wealings rats. Canad. J. anim. Sci., 38, r87-193.

BowLAND J. P., 1964. The relationship of energy and proteins in the diet of the growth and energy efficiency of young pigs. Ann. Zootech., 13, I4I-153.

Bosshard D. K., Winifred P., O'Doherty Katleen, Barnes R. H., 1946. The influence of caloric intake on the growth utilization of dietary protein. J. of Nulr., 32, 64I-65I.

BRODY S., 1945. Bioenergetics and growth. New York Reinhold.

CALET C., 1961. Étude de quelques facteurs nutritionnels qui retentissent sur l'appétit des volailles. Journées scientifiques de l' Aviculture. Paris. Compte rendu, 37-57.

Clawson A. J., Blumer T. N., SMART W. W. G., Barrick E. R., Ig62. Influence of energy protein ratio on performance and carcass characteristics of swine. J. anim. Sci., 21, 62-68.

FÉvrier R., 1959. Influence de l'alimentation sur l'importance des réserves grasses du Porc. Ann. de la Nutrition et de l'Alimentation, vol. 13, no 1,65 - 110.

Forbes E. B., Braman W. W., KrISS M., I93I. Further studies of energy metabolism of cattle in relation to the plane of nutrition. J. agric. Research., 40,37-78.

Forbes R. M., YoHE Hartha., I955. Effect of energy intake on the biological value of protein fed to rats. J. of Nutr., 55, 499-506.

GUILlAume J., I96I. Les facteurs de variations du rapport calories/matières azotées totales dans les régimes du poussin. Ann. Zootech., 10, 279-31 I.

HAMmOND J., 1932. Growth and the developpement of mutton qualities in the sheep. Oliver and Boyd. Edinburg.

HENRY Y., Rérat A., I963. Étude de l'ingestion spontanée d'éléments énergétiques et de protéines chez le rat en croissance par la méthode du libre choix. Ann. Biol. anim. Biophys., 3, 103-117.

HENRY Y., RÉrat A., 1964. Variations des taux énergétique et azoté dans l'alimentation d'un porc en croissance. Ann. Biol. anim. Biophys., 4, 263-271.

Kuryvial M. S., Bowland J. P., BerG R. T., 1962. Supplement fat as an energy source in the diets of swine and rats. Rood and Energy utilization. Canad. J. anim. Sci., 42, 23-32.

LikUSKI H. J. A., Bowland J. P., BERG R. T., 196I. Energy digestibility and nitrogen retention by pigs and rats fed diets contaning non nutritive diluents and varying protein level. Canad. J.anim. Sci., 41, 89-10I.

McMeEkan C. P., 1940. Growth and development in the pig with special reference to carcass quality characteristics. J.' Agric. Sci., 30, $276-344,387-436,5$ I 1-569.

McMeEKan C. P., I94!. Growth and development in the pig with special reference to carcass quality characteristics. J. Agric. Sci., 31, I-49.

Meyer J. H., Nelson A. O., 1963. Efficiency of feed utilization by various animals species fed similar rations. J. of Nutr., 80, 343-349.

NICKELSEN O., ANDERSON A. A., 1959. A method for preparing intact animals for carcass analyses. $J$. Lab. Clin. Med., 53, 2822-9o. 
Morin-Jomain M., I962. Le comportement nutritionnel du rat soumis à des restrictions alimentaires périodiques suivies de réalimentation. Arch. Sci. Physiol., 16, 44 I-456.

Morin-Jomain M., Abraham J., JacQuot R., ig64. Chronologie des apports alimentaires et capacité de protéinogenèse et de lipdogenèse chez le rat en croissance : études des régimes croisés, riches et pauvres en protéines. C. R. Acad. Sci., 258, I626-163.

Pond W. G., Krong E., Loosli J. K., 2960. Effect of level of dietary fat, panthotenic acid and protéin on performance of growing fattening swine. J. anim. Sci., 19, I1 15-1122.

Rerat A., Février C., Henry Y., Lougnon J., ig64. Evolution de la composition corporelle chez le rat blanc en croissance. Ann. Biol. anim. Biophys., 4, 35-47.

Rerat A., Henry Y., ig64. Étude du besoin azoté du Porc en croissance. Utilisation de la farine de poisson à trois taux azotés différents. Ann. Zootech., 13, 5-34.

Robinson D. W., Morgan J. T., Lewis D., r964. Protein and energy nutrition of the bacon pigs. $J$. of Agric. Sci., 63, 409-414.

Summers J. D., Slinger J. J., Sibbald I. R., Pepser W. F., 1964. Influence of protein and energy on growth and protein utilization in the growing chicken. J. of Nutr., 82, 463-468.

Summers J. D., Slinger J. J., Ashion G. C., I 965 . The effect of dietary energy and protein on carcass composition with a note of the method for estimating carcass composition poultry. Sci., 44, 50I-509.

Terroine E. F., Valla S., 1933. La valeur comparée des différents aliments protéiques dans la croissance. C. R. Acad. Sci., 196, 288-29o.

Wagner G. R., Clark A. J., Hays V. W., Speer V. C., 1963. Effect of protein energy relationship on the performance and carcass quality of growing swine. J.anim. Sci., 22, 202-208.

WALlACE H. D., I964. Dietary protein level, feed restriction and sex influences on the feedlot performance and carcass characteri stics of finishing swine. Feedstuffs, 37, 18-19.

Wilson P. N., Osbourn D. F., I960. Compensatory growth after undernutrition in mammals and birds. Biol. Reviews., 35, 324-363. 